\title{
A multi-parametric programming approach for multilevel hierarchical and decentralised optimisation problems
}

\author{
Nuno P. Faísca • Pedro M. Saraiva • Berç Rustem • \\ Efstratios N. Pistikopoulos
}

(C) Springer-Verlag 2007

\begin{abstract}
In this paper, we outline the foundations of a general global optimisation strategy for the solution of multilevel hierarchical and general decentralised multilevel problems, based on our recent developments on multi-parametric programming and control theory. The core idea is to recast each optimisation subproblem, present in the hierarchy, as a multi-parametric programming problem, with parameters being the optimisation variables belonging to the remaining subproblems. This then transforms the multilevel problem into single-level linear/convex optimisation problems. For decentralised systems, where more than one optimisation problem is present at each level of the hierarchy, Nash equilibrium is considered. A three person dynamic optimisation problem is presented to illustrate the mathematical developments.
\end{abstract}

Keywords Hierarchical decision making - Multilevel programming ·

Multi-parametric programming $\cdot$ Discrete-time systems $\cdot$ Closed-loop optimal control

\section{Introduction}

The development of a general theory to solve multi-person objective decision problems is of great importance for decision making and control theory (Başar 1975). Multi-person objective decision problems have attracted numerous investigations

\footnotetext{
N. P. Faísca · B. Rustem · E. N. Pistikopoulos ( $\varangle)$

Centre for Process Systems Engineering,

Imperial College London, London SW7 2AZ, UK

e-mail: e.pistikopoulos@imperial.ac.uk

P. M. Saraiva

Gepsi-PSE Group, Department of Chemical Engineering,

University of Coimbra, 3030-290 Coimbra, Portugal
} 
Fig. 1 Hierarchical control of an automatic vehicle (Rodić and Vukobratović 1999)
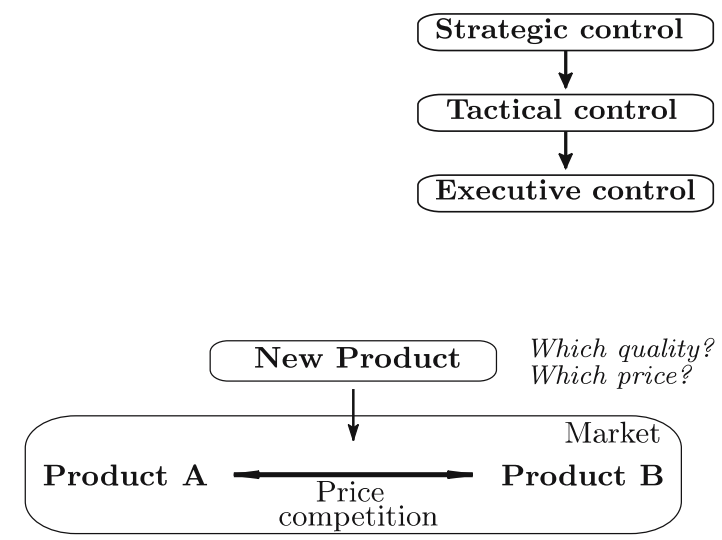

competition
Fig. 2 Product positioning under price competition (Choi et al. 1990)

(Başar 1975, 1978; Tolwinski 1981; Başar and Olsder 1982; Anandalingman 1988; Liu 1998; Li et al. 2002; Shih et al. 2004), with diverse applications in engineering (Morari et al. 1980; Clark 1983; Stephanopoulos and Ng 2000), financial problems (Anandalingman 1988; Nie et al. 2006) and in other areas, two examples of such applications are depicted in Figs. 1 and 2.

In this work we focus on multilevel decentralised optimisation problems, where the objectives (optimisation subproblems) are organised in a hierarchy of decisions. In this hierarchy, each optimisation subproblem controls a subset of the full set of optimisation variables; the latter is completely controlled by the unique optimisation problem positioned at the top level.

The multi-layer nature in such problems results in non-linearities and non-convexities (Vicente and Calamai 1994); hence, it is not surprising that general solution strategies for solving such complex problems are rather limited. Moreover, the possible presence of logical decisions further increases the problems' complexity. Therefore, it is widely accepted that a global optimisation approach is needed for the solution of such multilevel problems (Floudas 2000).

Recently, Pistikopoulos and co-workers have been developing a general theory, algorithms and computation tools for the solution of general classes of multi-parametric programming problems (Pistikopoulos et al. 2007a) and multi-parametric control (Pistikopoulos et al. 2007b). The application of parametric programming theory to multi-level problems (Faísca et al. 2007b) makes possible the development of a unified strategy for their solution to global optimality. The core idea behind this approach is to recast each optimisation subproblem as a multi-parametric programming problem. Computing the rational reaction set for each subproblem in the entire feasible space, and subsequently, computing the corresponding equilibria within the hierarchical network, disassembles the complexity of the original problem. For instance, in an optimisation level with two subproblems or more, these explicit expressions are used to compute the Nash equilibrium between them. In our previous work (Faísca et al. 2007b; Pistikopoulos et al. 2007a) we have addressed the bilevel programming problem, a hierarchy of two optimisation subproblems organised in two levels. In this paper we extend the methodology proposed in Faísca et al. (2007b) to cope with multilevel 
decentralised optimisation problems. Furthermore, the methodology is applied to an optimal control problem of multi-level nature, where the foundations of a general theory for multi-level hierarchical and decentralised problems are established.

This paper is organised as follows. Section 2 introduces the multi-level mathematical formulation, which is used throughout the paper, and respective definitions of feasible and rational reaction set. It also briefly introduces the relevant multi-parametric programming theory and algorithms. The proposed multi-parametric programming approach for the solution of tri-level programming problems and bilevel programming with multi-followers problems is then described in detail in Sect. 3, and illustrated with example problems. Sect. 4 outlines the application of the proposed approach to multilevel optimal control of dynamic systems.

\section{Preliminaries}

\subsection{Problem formulation}

The general multilevel decentralised optimisation problem can be described as follows:

$$
\begin{gathered}
\min _{x, y_{1}^{i}, y_{2}^{k}, \ldots, y_{m}^{l}} f_{1}\left(x, y_{1}^{i}, y_{2}^{k}, \ldots, y_{m}^{l}\right), \quad \text { (1st level) } \\
\text { s.t. } g_{1}\left(x, y_{1}^{i}, y_{2}^{k}, \ldots, y_{m}^{l}\right) \leq 0, \\
\text { where }\left[y_{1}^{i}, y_{2}^{k}, \ldots, y_{m}^{l}\right] \text { solve, } \\
\ldots, \min _{y_{1}^{i}, y_{2}^{k}, \ldots, y_{m}^{l}} f_{2}^{i}\left(x, y_{1}^{i}, y_{2}^{k}, \ldots, y_{m}^{l}\right), \ldots \quad \text { (2nd level) } \\
\text { s.t. } g_{2}^{i}\left(x, y_{1}^{i}, y_{2}^{k}, \ldots, y_{m}^{l}\right), \leq 0, \\
\text { where }\left[y_{2}^{k}, \ldots, y_{m}^{l}\right] \text { solve, } \\
\vdots \quad \ldots, \min _{y_{m}^{l}} f_{m}^{l}\left(x, y_{1}^{i}, y_{2}^{k}, \ldots, y_{m}^{l}\right), \ldots \text { (mth level) } \\
\text { s.t. } g_{m}^{l}\left(x, y_{1}^{i}, y_{2}^{k}, \ldots, y_{m}^{l}\right) \leq 0 .
\end{gathered}
$$

Here, $f$ are real convex functions, $g$ are vectorial real functions defining convex sets and $x, y$ are sets of variables belonging to the group of real numbers; $i \in$ $\{1,2, \ldots, I\}, k \in\{1,2, \ldots, K\}, l \in\{1,2, \ldots, L\}$, implying that (2nd level) has $I$ optimisation subproblems (3rd level) $K$ optimisation subproblems and ( $m$ th level) has $L$ optimisation subproblems, respectively.

For the sake of simplicity and without loss of generality, we analyse the relations in Problem (1) using two particular classes of multilevel programming problems: the trilevel programming problem, which organises vertically in three levels, and the bilevel programming problem with multi-followers, in a horizontal structure at the second level. 


\subsubsection{Tri-level programming}

The tri-level programming problem can be stated as follows:

$$
\begin{aligned}
& \min _{x, y_{1}, y_{2}} f_{1}\left(x, y_{1}, y_{2}\right), \quad \text { (1st level) } \\
& \text { s.t. } g_{1}\left(x, y_{1}, y_{2}\right) \leq 0 \text {, } \\
& \text { where }\left[y_{1}, y_{2}\right] \text { solve, } \\
& \min _{y_{1}, y_{2}} f_{2}\left(x, y_{1}, y_{2}\right), \quad \text { (2nd level) } \\
& \text { s.t. } g_{2}\left(x, y_{1}, y_{2}\right), \leq 0 \text {, } \\
& \text { where }\left[y_{2}\right] \text { solve, } \\
& \min _{y_{2}} f_{3}\left(x, y_{1}, y_{2}\right), \quad \text { (3rd level) } \\
& \text { s.t. } g_{3}\left(x, y_{1}, y_{2}\right) \leq 0 \text {, }
\end{aligned}
$$

with the following definitions:

- feasible set for the third level,

$$
\Omega_{2}\left(x, y_{1}\right)=\left\{y_{2} \in Y_{2}: g_{3}\left(x, y_{1}, y_{2}\right) \leq 0\right\},
$$

- rational reaction set for the third level,

$$
\phi_{2}\left(x, y_{1}\right)=\left\{y_{2} \in Y_{2}: y_{2} \in \operatorname{argmin}\left\{f_{2}\left(x, y_{1}, y_{2}\right): y_{2} \in \Omega_{2}\left(x, y_{1}\right)\right\}\right\},
$$

- feasible set for the second level,

$$
\Omega_{1}(x)=\left\{y_{1}, y_{2} \in Y_{1}, Y_{2}: g_{2}\left(x, y_{1}, y_{2}\right) \leq 0, g_{3}\left(x, y_{1}, y_{2}\right) \leq 0\right\}
$$

- rational reaction set for the second level,

$$
\begin{aligned}
\phi_{1}(x)= & \left\{y_{1}, y_{2} \in Y_{1}, Y_{2}: y_{1} \in \operatorname{argmin}\right. \\
& \left.\times\left\{f_{2}\left(x, y_{1}, y_{2}\right): y_{1} \in \Omega_{1}(x), y_{2} \in \phi_{2}\left(x, y_{1}\right)\right\}\right\} .
\end{aligned}
$$

Note the parametric nature of the rational reaction sets, Eqs. (4) and (6), which reflects the dependence of the decisions taken at the upper levels on the decisions taken at the lower levels. This in fact, evidences that in multilevel programming problems the relations between the levels differ from the well-known Stackelberg game, where the decisions made by the followers don't affect the decision, already taken by the leader (Vicente 1992). 


\subsubsection{Bilevel programming with multi-followers}

Bilevel programming problems with multi-followers involve two optimisation levels with several optimisation subproblems at the lower (2nd) level:

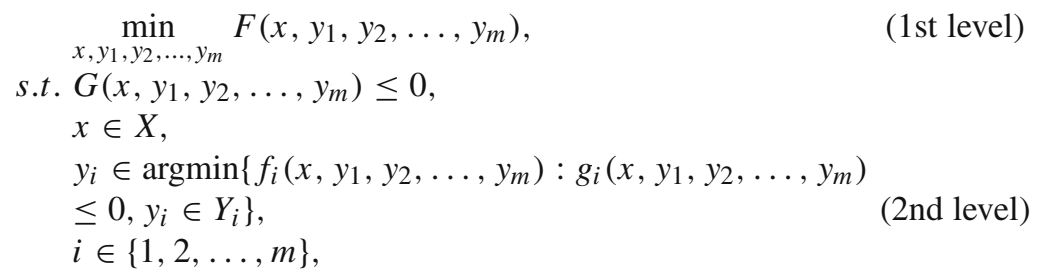

with the following definitions:

- feasible set for the $i$ th follower,

$$
\Omega_{i}\left(x, y_{1}, y_{2}, \ldots, y_{i-1}, y_{i+1}, \ldots, y_{m}\right)=\left\{y_{i} \in Y_{i}: g_{i}\left(x, y_{1}, y_{2}, \ldots, y_{m}\right) \leq 0\right\},
$$

- rational reaction set for the $i$ th follower,

$$
\begin{aligned}
& \phi_{i}\left(x, y_{1}, y_{2}, \ldots, y_{i-1}, y_{i+1}, \ldots, y_{m}\right) \\
& \quad=\left\{y_{i} \in Y_{i}: y_{i} \in \operatorname{argmin}\left\{f_{i}\left(x, y_{1}, y_{2}, \ldots, y_{m}\right): y_{i} \in \Omega_{i}(x)\right\}\right\} .
\end{aligned}
$$

Since one assumption is that followers may exchange information, conflicts naturally occur. The Nash equilibrium is often a preferred strategy to coordinate such decentralised systems (Başar 1975, 1978; Cruz 1978; Başar and Selbuz 1979; Choi et al. 1990; Liu 1998). Consequently, the optimisation subproblems positioned in the lower level are assumed to reach a Nash equilibrium point $\left(x, y_{1}^{*}, y_{2}^{*}, \ldots, y_{m}^{*}\right)$ (Başar and Olsder 1982):

$$
\left\{\begin{array}{l}
f_{1}\left(x, y_{1}^{*}, y_{2}^{*}, \ldots, y_{m}^{*}\right) \leq f_{1}\left(x, y_{1}, y_{2}^{*}, \ldots, y_{m}^{*}\right), \forall y_{1} \in Y_{1}, \\
f_{2}\left(x, y_{1}^{*}, y_{2}^{*}, \ldots, y_{m}^{*}\right) \leq f_{2}\left(x, y_{1}^{*}, y_{2}, \ldots, y_{m}^{*}\right), \forall y_{2} \in Y_{2}, \\
\vdots \\
f_{m}\left(x, y_{1}^{*}, y_{2}^{*}, \ldots, y_{m}^{*}\right) \leq f_{m}\left(x, y_{1}^{*}, y_{2}^{*}, \ldots, y_{m}\right), \forall y_{m} \in Y_{m} .
\end{array}\right.
$$

Once more observe the parametric nature of the followers' rational reaction set, Eq. (9). In this case, however, each rational reaction set is a function of both the upper level decision variables and the decision variables of the other subproblems located in the same hierarchical level. Additionally, the priority remains to solve the leader's objective function to global optimality. Thus, we aim to compute the set $\left\{x, y_{1}, \ldots, y_{m}\right\}$ which optimises globally the leader objective:

$$
\min _{x, y_{1}, \ldots, y_{m}}\left\{F\left(x, y_{1}, \ldots, y_{m}\right): G\left(x, y_{1}, \ldots, y_{m}\right) \leq 0, y_{i} \in \phi_{i}, i=1, \ldots, m\right\},
$$

and the set $\left\{y_{1}, \ldots, y_{m}\right\}$ which corresponds to a Nash equilibrium point, Eq. (10). 


\subsection{Multi-parametric programming}

Consider the general multi-parametric non-linear programming problem:

$$
\begin{array}{ll} 
& \min _{x} f(x, \theta), \\
\text { s.t. } & g_{i}(x, \theta) \leq 0, \quad \forall i=1, \ldots, p, \\
& h_{j}(x, \theta)=0, \quad \forall j=1, \ldots, q, \\
& x \in X \subseteq \mathbb{R}^{n}, \\
& \theta \in \Theta \subseteq \mathbb{R}^{m},
\end{array}
$$

where $f, g$ and $h$ are twice continuously differentiable in $x$ and $\theta$. Assume also that $f$ is a convex function and $g, h$ define a convex set. Therefore, the first-order KarushKuhn-Tucker (KKT) optimality conditions for (12) are given as follows:

$$
\begin{aligned}
& \mathscr{L}=f(x, \theta)+\sum_{i=1}^{p} \lambda_{i} g_{i}(x, \theta)+\sum_{j=1}^{q} \mu_{j} h_{j}(x, \theta), \\
& \nabla_{x} \mathscr{L}=0, \\
& \lambda_{i} g_{i}(x, \theta)=0, \quad \lambda_{i} \geq 0, \quad \forall i=1, \ldots, p, \\
& h_{j}(x, \theta)=0, \quad \forall j=1, \ldots, q .
\end{aligned}
$$

The main sensitivity result for (12) derives directly from system (13), as shown in Theorem 1.

Theorem 1 Basic sensitivity theorem (Fiacco 1976): let $\theta_{0}$ be a vector of parameter values and $\left(x_{0}, \lambda_{0}, \mu_{0}\right)$ a KKT triple corresponding to (13), where $\lambda_{0}$ is nonnegative and $x_{0}$ is feasible in (12). Also assume that (i) strict complementary slackness (SCS) holds, (ii) the binding constraint gradients are linearly independent (LICQ: Linear Independence Constraint Qualification), and (iii) the second-order sufficiency conditions (SOSC) hold. Then, in the neighbourhood of $\theta_{0}$, there exists a unique, once continuously differentiable function, $z(\theta)=[x(\theta), \lambda(\theta), \mu(\theta)]$, satisfying (13) with $z\left(\theta_{0}\right)=\left[x\left(\theta_{0}\right), \lambda\left(\theta_{0}\right), \mu\left(\theta_{0}\right)\right]$, where $x(\theta)$ is a unique isolated minimiser for (12), and

$$
\left(\begin{array}{l}
\frac{d x\left(x_{0}\right)}{d \theta} \\
\frac{d \lambda\left(x_{0}\right)}{d \theta} \\
\frac{d \mu\left(x_{0}\right)}{d \theta}
\end{array}\right)=-\left(M_{0}\right)^{-1} N_{0},
$$

where, $M_{0}$ and $N_{0}$ are the Jacobian of system (13) with respect to $z$ and $\theta$ :

$$
M_{0}=\left(\begin{array}{c|cc|c}
\nabla_{x x}^{2} \mathscr{L} & \nabla_{x} g_{1} \cdots \nabla_{x} g_{p} & \nabla_{x} h_{1} \cdots \nabla_{x} h_{q} \\
\hline-\lambda_{1} \nabla_{x}^{T} g_{1} & -g_{1} & & \\
\vdots & & \ddots & 0 \\
-\lambda_{p} \nabla_{x}^{T} g_{p} & & -g_{p} & \\
\hline \nabla_{x}^{T} h_{1} & & \\
\vdots & & 0 & 0 \\
\nabla_{x}^{T} h_{q} & & &
\end{array}\right),
$$




$$
N_{0}=\left(\nabla_{\theta x}^{2} \mathscr{L},-\lambda_{1} \nabla_{\theta}^{T} g_{1}, \ldots,-\lambda_{p} \nabla_{\theta}^{T} g_{p}, \nabla_{\theta}^{T} h_{1}, \ldots, \nabla_{\theta}^{T} h_{q}\right)^{T} .
$$

Proof See (Fiacco 1983, pp 72).

Note that the assumptions stated in the theorem above ensure $M_{0}$ is invertible (McCormick 1976).

Dua et al. (2002) has proposed an algorithm to solve Eq. (14) in the entire range of the varying parameters for general convex problems. This algorithm is based on approximations of the non-linear optimal expression, $x=\gamma^{*}(\theta)$, by a set of first-order approximations (Corollary 1).

Corollary 1 First-order estimation of $x(\theta), \lambda(\theta), \mu(\theta)$, near $\theta=\theta_{0}$ (Fiacco 1983): Under the assumptions of Theorem 1, a first-order approximation of $[x(\theta), \lambda(\theta)$, $\mu(\theta)]$ in the neighbourhood of $\theta_{0}$ is,

$$
\left[\begin{array}{l}
x(\theta) \\
\lambda(\theta) \\
\mu(\theta)
\end{array}\right]=\left[\begin{array}{l}
x_{0} \\
\lambda_{0} \\
\mu_{0}
\end{array}\right]-\left(M_{0}\right)^{-1} \cdot N_{0} \cdot \theta+o(\|\theta\|),
$$

where $\left(x_{0}, \lambda_{0}, \mu_{0}\right)=\left[x\left(\theta_{0}\right), \lambda\left(\theta_{0}\right), \mu\left(\theta_{0}\right)\right], M_{0}=M\left(\theta_{0}\right), N_{0}=N\left(\theta_{0}\right)$, and $\phi(\theta)=$ $o(\|\theta\|)$ means that $\phi(\theta) /\|\theta\| \rightarrow 0$ as $\theta \rightarrow \theta_{0}$.

Each piecewise linear approximation is confined to regions defined by feasibility and optimality conditions (Dua et al. 2002). If $\breve{g}$ corresponds to the non-active constraints, and $\tilde{\lambda}$ to the Lagrangian multipliers of the active constraints:

$$
\begin{cases}\breve{g}(x(\theta), \theta) \leq 0 & \rightarrow \text { Feasibility conditions } \\ \tilde{\lambda}(\theta) \geq 0 & \rightarrow \text { Optimality conditions }\end{cases}
$$

Consequently, the explicit expressions are given by a conditional piecewise linear function (Dua et al. 2002):

$$
\left\{\begin{array}{c}
x=\mathbf{C}^{1}+\mathbf{K}^{1} \cdot \theta, \quad \theta \in C R^{1}, \\
x=\mathbf{C}^{2}+\mathbf{K}^{2} \cdot \theta, \quad \theta \in C R^{2}, \\
\vdots \\
x=\mathbf{C}^{L}+\mathbf{K}^{L} \cdot \theta, \theta \in C R^{L},
\end{array}\right.
$$

where $\mathbf{K}^{i}$ and $\mathbf{C}^{i}$ are real matrices, and $C R^{i} \subset \mathbb{R}^{m}$.

\section{Proposed methodology}

In this section, we show how we can address tri-level programming and bilevel programming with multi-followers problems, and solve them to global optimality through the application of parametric programming. For the sake of clarity, the methodology is described using formulations with quadratic cost functions and linear constraints, however, it is applicable to general non-linear problems using suitable multi-parametric programming algorithms (Dua et al. 2004). 


\subsection{Tri-level programming problem}

Consider the tri-level programming problem with a quadratic objective function and linear constraints:

$$
\begin{aligned}
& \min _{x, y_{1}, y_{2}} f_{1}=L_{1}^{1} \\
& +L_{2}^{1} \cdot x+L_{3}^{1} \cdot y_{1}+L_{4}^{1} \cdot y_{2} \\
& +\frac{1}{2} x^{T} \cdot L_{5}^{1} \cdot x+\frac{1}{2} y_{1}^{T} \cdot L_{6}^{1} \cdot y_{1}+\frac{1}{2} y_{2}^{T} \cdot L_{7}^{1} \cdot y_{2} \\
& +x^{T} \cdot L_{8}^{1} \cdot y_{1}+y_{2}^{T} \cdot L_{9}^{1} \cdot x+y_{2}^{T} \cdot L_{10}^{1} \cdot y_{1} \text {, } \\
& \mid \begin{array}{l}
G_{1}^{1} \cdot x+G_{2}^{1} \cdot y_{1}+G_{3}^{1} \cdot y_{2} \leq 0, \\
\min _{y_{1}, y_{2}} f_{2}=L_{1}^{2}
\end{array} \\
& +L_{2}^{2} \cdot x+L_{3}^{2} \cdot y_{1}+L_{4}^{2} \cdot y_{2} \\
& +\frac{1}{2} x^{T} \cdot L_{5}^{2} \cdot x+\frac{1}{2} y_{1}^{T} \cdot L_{6}^{2} \cdot y_{1}+\frac{1}{2} y_{2}^{T} \cdot L_{7}^{2} \cdot y_{2} \quad \text { (2nd level) } \\
& +x^{T} \cdot L_{8}^{2} \cdot y_{1}+y_{2}^{T} \cdot L_{9}^{2} \cdot x+y_{2}^{T} \cdot L_{10}^{2} \cdot y_{1} \text {, } \\
& \begin{array}{l|l}
\text { s.t. } & G_{1}^{2} \cdot x+G_{2}^{2} \cdot y_{1}+G_{3}^{2} \cdot y_{2} \leq 0,
\end{array} \\
& \min _{y_{2}} f_{3}=L_{1}^{3} \\
& \begin{array}{ll}
\quad y_{2} \quad & +L_{2}^{3} \cdot x+L_{3}^{3} \cdot y_{1}+L_{4}^{3} \cdot y_{2} \quad(3 \mathrm{rd} \text { level }) \\
\text { s.t. } & +\frac{1}{2} x^{T} \cdot L_{5}^{3} \cdot x+\frac{1}{2} y_{1}^{T} \cdot L_{6}^{3} \cdot y_{1}+\frac{1}{2} y_{2}^{T} \cdot L_{7}^{3} \cdot y_{2}
\end{array} \\
& +x^{T} \cdot L_{8}^{3} \cdot y_{1}+y_{2}^{T} \cdot L_{9}^{3} \cdot x+y_{2}^{T} \cdot L_{10}^{3} \cdot y_{1} \text {, } \\
& \text { s.t. } \mid G_{1}^{3} \cdot x+G_{2}^{3} \cdot y_{1}+G_{3}^{3} \cdot y_{2} \leq 0 \text {. }
\end{aligned}
$$

Problem (18) comprises three subproblems, one at each optimisation level. Each optimisation level can be recast as a multi-parametric programming problem, where the optimisation variables corresponding to the upper optimisation levels are classified as parameters. For presentation and computation purposes, (i) we group the parameters in the $i$ th level in a single vector, $\omega^{i}$ and (ii) we introduce an artificial variable, $v^{i}$, to eliminate all bilinear terms.

Beginning with the (3rd level), and considering a vector,

$$
\left[\omega^{3}\right]^{T}=\left[x \mid y_{1}\right]
$$

we re-write (18) as,

$$
\begin{gathered}
\min _{y_{2}} f_{3}\left(y_{2}, \omega^{3}\right)= \\
L_{1}^{3}+L_{2}^{3 *} \cdot \omega^{3}+L_{4}^{3} \cdot y_{2}+\frac{1}{2} \omega^{3^{T}} \cdot L_{5}^{3 *} \cdot \omega^{3} \\
\quad+\frac{1}{2} y_{2}^{T} \cdot L_{7}^{3} \cdot y_{2}+y_{2}^{T} \cdot L_{8}^{3 *} \cdot \omega^{3}, \\
\text { s.t. } G_{1}^{3 *} \cdot \omega^{3}+G_{3}^{3} \cdot y_{2}+G_{4}^{3} \leq 0, \quad x \in X .
\end{gathered}
$$

Introducing an artificial variable, $v^{3}=y_{2}+\Phi \cdot \omega^{3}$, where $\Phi$ is an appropriate matrix, the bilinear terms, represented in (19) by matrix $L_{8}^{3 *}$, are eliminated. Under the right conditions (Faísca et al. 2007b), $\Phi=L_{7}^{3^{-1}} L_{8}^{3 *}$, and (19) can be rewritten as follows: 


$$
\begin{aligned}
\min _{v_{3}} f_{3}\left(v_{3}, \omega^{3}\right)= & L_{1}^{3}+L_{2}^{3 * *} \cdot \omega^{3}+\frac{1}{2} \omega^{3^{T}} \cdot L_{5}^{3 * *} \cdot \omega^{3} \\
& +\min _{v_{3}}\left\{L_{4}^{3 * *} \cdot v_{3}+\frac{1}{2} v_{3}^{T} \cdot L_{7}^{3 * *} \cdot v_{3}\right\}, \\
\text { s.t. } G_{3}^{3 * *} \cdot v_{3} \leq & G_{4}^{3 * *}+G_{1}^{3 * *} \cdot \omega^{3}, v \in V,
\end{aligned}
$$

Problem (20) can be solved with a multi-parametric programming algorithm (Dua et al. 2002), resulting in:

$$
v_{3}^{k}=m_{3}^{k}+n_{3}^{k} \cdot \omega^{3}, \quad H_{3}^{k} \cdot \omega^{3} \leq h_{3}^{k},
$$

which can be rewritten as,

$$
\begin{aligned}
& y_{2}^{k}=m_{3}^{k}+\left(n_{3}^{k}-\Phi\right) \cdot \omega^{3}, \quad H_{3}^{k} \cdot \omega^{3} \leq h_{3}^{k}, \\
& \text { or, } \\
& y_{2}^{k}=m_{3}^{k}+p_{1}^{k} \cdot x+p_{2}^{k} \cdot y_{1}, \quad H_{31}^{k} \cdot x+H_{32}^{k} \cdot y_{1} \leq h^{k},
\end{aligned}
$$

where, $k=1, \ldots, K_{2}$, with $K_{2}$ being the number of critical region, and consequently, the number of linear approximations done on the optimal rational reaction set $\phi_{2}\left(x, y_{1}\right)$ (see Corollary 1$)$.

The expressions in (21) can then be incorporated in the second optimisation level of (18). Note that since the expressions in (21) are piecewise linear functions of $y_{2}^{k}$, the complexity of the original problem does not increase. Hence, the second level can be reformulated as the following $K_{2}$ optimisation problems:

$$
\begin{aligned}
& \min _{y_{1}} f_{2}=L_{1}^{2 *}+L_{2}^{2 *} \cdot x+L_{3}^{2 *} \cdot y_{1}+\frac{1}{2} x^{T} \cdot L_{4}^{2 *} \cdot x+\frac{1}{2} y_{1}^{T} \cdot L_{5}^{2 *} \cdot y_{1}+y_{1}^{T} \cdot L_{8}^{2 *} \cdot x, \\
& \text { s.t. } G_{1}^{2 *} \cdot x+G_{2}^{2 *} \cdot y_{1}+G_{3}^{2 *} \leq 0, x \in X .
\end{aligned}
$$

We can thus proceed with optimisation levels 1 and 2. Following this procedure, tri-level optimisation problems in (18) result in $K_{1}$ single level convex optimisation problems:

$$
\begin{array}{ll} 
& \min _{x} f_{1}^{*}\left(x, y_{1}(x), y_{2}\left(x, y_{1}\right)\right), \\
\text { s.t. } & G_{1}\left(x, y_{1}(x), y_{2}\left(x, y_{1}(x)\right)\right) \leq 0, \\
& x \in C_{r f}, \\
& C_{r f}=\left\{x \in X: \exists_{y_{1}, y_{2}} \in Y_{1}, Y_{2}, G_{2}\left(x, y_{1}, y_{2}\right) \leq 0, G_{3}\left(x, y_{1}, y_{2}\right) \leq 0\right\} .
\end{array}
$$

The number of $K_{1}$ final convex optimisation problems (23) depends on the number of critical regions obtained in each optimisation level. The algorithm is summarised in Table 1, and is illustrated with the following example. 
Table 1 Parametric programming algorithm for tri-level programming problems

\begin{tabular}{|c|c|}
\hline Step & Description \\
\hline 1 & $\begin{array}{l}\text { Recast the third level of the optimisation } \\
\text { problem as a multi-parametric programming } \\
\text { problem, with parameters being the upper } \\
\text { levels optimisation variables, } x \text { and } y_{1}(19)\end{array}$ \\
\hline 2 & $\begin{array}{l}\text { Solve the resulting problem using a suitable } \\
\text { multi-parametric programming algorithm }\end{array}$ \\
\hline
\end{tabular}

Substitute each of the $K_{2}$ solutions in the 2nd optimisation level, and formulate $K_{2}$ multiparametric problems with the variables from the leader being the parameters (22)

4

Solve the resulting problem using a suitable multi-parametric programming algorithm

Substitute each of the $K_{1}$ solutions in the leader's problem, and formulate the $K_{1}$ onelevel optimisation problems (23) one

\subsubsection{Illustrative example 1}

Consider the following linear tri-level example (Ruan et al. 2004):

$$
\begin{aligned}
& \min _{x, y_{1}, y_{2}} f_{1}=-x-4 \cdot y_{2}, \\
& \text { where }\left[y_{1}, y_{2}\right] \text { solve, } \\
& \min _{y_{1}, y_{2}} f_{2}=2 \cdot y_{2}, \\
& \text { where } y_{2} \text { solves, } \\
& \min _{y_{2}} f_{3}=-y_{2}, \\
& \text { s.t. } x+y_{1}+y_{2} \leq 2.5, \\
& 0 \leq x, y_{1}, y_{2} \leq 1 .
\end{aligned}
$$

Following the steps described in Table 1:

Step 1. Recast (3rd) level optimisation problem, $f_{3}$, as a multi-parametric programming problem, with parameters being $x$ and $y_{1}$

$$
\begin{gathered}
\min _{y_{2}} f_{3}=-y_{2}, \\
\text { s.t. } y_{2} \leq 2.5-x-y_{1}, \\
0 \leq x, y_{1}, y_{2} \leq 1,
\end{gathered}
$$

solve the resulting problem using a multi-parametric optimisation algorithm (Dua et al. 2002):

$$
C R^{1}\left\{\begin{array} { l } 
{ y _ { 2 } = 1 } \\
{ 0 \leq x , y _ { 1 } \leq 1 , } \\
{ x + y _ { 1 } \leq 1 . 5 , }
\end{array} \quad C R ^ { 2 } \left\{\begin{array}{l}
y_{2}=-x-y_{1}+2.5 \\
x, y_{1} \leq 1 \\
-x-y_{1} \leq-1.5
\end{array}\right.\right.
$$


Step 2. Incorporate rational reaction set (26) into the optimisation problem corresponding to (2nd) level;

$$
\begin{array}{ll}
\min _{y_{1}, y_{2}} f_{2}^{C R^{1}}=2, & \min _{y_{1}, y_{2}} f_{2}^{C R^{2}}=-2 x-2 y_{1}+5, \\
\text { s.t. } 0 \leq x \leq 1, & \text { s.t. } x, y_{1} \leq 1, \\
0 \leq y_{1} \leq 1, & -x-y_{1} \leq-1.5 . \\
x+y_{1} \leq 1.5, &
\end{array}
$$

Step 3. Solve problems (27) considering them as multi-parametric programming problems, with $x$ being parameter;

$$
C R^{3}\left\{\begin{array} { l } 
{ y _ { 2 } = 1 } \\
{ 0 \leq x \leq 1 , } \\
{ 0 \leq y _ { 1 } \leq 1 , } \\
{ x + y _ { 1 } \leq 1 . 5 , }
\end{array} \quad C R ^ { 4 } \left\{\begin{array}{l}
y_{1}=1 \\
y_{2}=-x+1.5 \\
0.5 \leq x \leq 1
\end{array}\right.\right.
$$

Step 4. Incorporate rational reaction set (28) into the optimisation problem corresponding to (1st) level;

$$
\begin{array}{ll}
\min _{x, y_{1}, y_{2}} f_{1}^{C R^{3}}=-x-4, & \\
\text { s.t. } 0 \leq x \leq 1, & \min _{x, y_{1}, y_{2}} f_{1}^{C R^{4}}=3 x-6, \\
0 \leq y_{1} \leq 1, & \text { s.t. } 0.5 \leq x \leq 1 \\
x+y_{1} \leq 1.5, &
\end{array}
$$

Step 5. Solve problems in (29);

$$
\text { Solution } 1\left\{\begin{array} { l } 
{ f _ { 1 } ^ { C R ^ { 3 } } = - 5 , } \\
{ x = 1 , } \\
{ y _ { 2 } = 1 , } \\
{ 0 \leq y _ { 1 } \leq 0 . 5 , }
\end{array} \quad \text { Solution } 2 \left\{\begin{array}{l}
f_{1}^{C R^{4}}=-4.5 \\
x=0.5 \\
y_{1}=1 \\
y_{2}=1
\end{array}\right.\right.
$$

Note that in Solution 1, $y_{1}$ is represented by an interval. This is due to the fact that the objective function of (2nd level) does not depend on $y_{1}$.

Concluding, two solutions are obtained: Solution 1 and Solution 2, which are compared with the one obtained from the literature (Ruan et al. 2004, Solution 3), as shown in Table 2.

From Table 2 we conclude that Solution 1 is the global optimum for this tri-level programming problem.

\subsection{Bilevel programming problem with multi-followers}

Consider the bilevel programming problem with multi-followers, and assume quadratic objective functions, linear constraints and two followers: 
Table 2 Solutions for problem (24)

\begin{tabular}{lllll}
\hline & \multicolumn{2}{l}{ Parametric programming algorithm } & & Ruan et al. (2004) \\
\cline { 2 - 3 } & Solution 1 & Solution 2 & & Solution 3 \\
\hline$f^{1}$ & -5 & -4.5 & -4.5 \\
$f^{2}$ & 2 & 2 & 2 \\
$f^{3}$ & 1 & 1 & 1 \\
$x$ & 1 & 0.5 & - \\
$y_{1}$ & 0.5 & 0 & - \\
$y_{2}$ & 1 & 0 & - \\
\hline
\end{tabular}

The difference between Problem (31) and Problem (18) is the existence of two optimisation subproblems in a single level. Accordingly, the concept of Nash equilibrium is introduced.

As in the tri-level programming case, each optimisation subproblem in (2nd) level is recast as a multi-parametric programming problem. In this problem, the parameters are all the variables from the optimisation problem at (1st) level as well as the optimisation variables of the other subproblems at the same level, Follower 1 or Follower 2 in this case (31). Thus, defining vectors, $\left[\omega^{2}\right]^{T}=\left[x \mid y_{2}\right]$ and $\left[\omega^{3}\right]^{T}=\left[x \mid y_{1}\right]$, we re-write the (2nd) level optimisation subproblems as,

$$
\begin{aligned}
& \min _{y_{1}} f_{2}\left(y_{1}, \omega^{2}\right)=L_{1}^{2}+L_{2}^{2 *} \cdot \omega^{2}+L_{3}^{2} \cdot y_{1}+\frac{1}{2} \omega^{2^{T}} \cdot L_{5}^{2 *} \cdot \omega^{2} \\
& +\frac{1}{2} y_{1}^{T} \cdot L_{6}^{2} \cdot y_{1}+y_{1}^{T} \cdot L_{8}^{2 *} \cdot \omega^{2} \\
& \text { s.t. } G_{1}^{2 *} \cdot \omega^{2}+G_{2}^{2} \cdot y_{1} \leq 0 \text {, }
\end{aligned}
$$


and,

$$
\begin{aligned}
\min _{y_{2}} f_{3}\left(y_{2}, \omega^{2}\right)= & L_{1}^{3}+L_{2}^{3 *} \cdot \omega^{3}+L_{4}^{3} \cdot y_{2}+\frac{1}{2} \omega^{3^{T}} \cdot L_{5}^{3 *} \cdot \omega^{2 a} \\
& +\frac{1}{2} y_{2}^{T} \cdot L_{7}^{3} \cdot y_{2}+y_{1}^{T} \cdot L_{9}^{3 *} \cdot \omega^{3}, \\
\text { s.t. } G_{1}^{3 *} \cdot \omega^{3}+ & G_{3}^{3} \cdot y_{2} \leq 0,
\end{aligned}
$$

where $\omega^{2}$ and $\omega^{3}$ are the vectors of parameters. The bi-linearities can be circumvented using a strategy similar to the one used in the tri-level case. Using a multi-parametric programming algorithm (Dua et al. 2002), problems (32) and (33) result in the following parametric expressions:

$$
\left\{\begin{array}{l}
y_{1}=\phi_{1}\left(x, y_{2}\right) \rightarrow \text { rational reaction set follower } 1 \\
y_{2}=\phi_{2}\left(x, y_{1}\right) \rightarrow \text { rational reaction set follower } 2
\end{array}\right.
$$

which are then used to compute the Nash equilibrium $\left(x, y_{1}^{*}, y_{2}^{*}\right)$ :

$$
\left\{\begin{array}{l}
f_{1}\left(x, y_{1}^{*}, y_{2}^{*}\right) \leq f_{1}\left(x, y_{1}, y_{2}^{*}\right), \forall y_{1} \in Y_{1} \\
f_{2}\left(x, y_{1}^{*}, y_{2}^{*}\right) \leq f_{2}\left(x, y_{1}^{*}, y_{2}\right), \forall y_{2} \in Y_{2}
\end{array}\right.
$$

easily computed by direct comparison (Liu 1998):

$$
\begin{aligned}
& \phi_{1}^{\prime}\left(x, y_{1}\right)=\phi_{2}\left(x, y_{1}\right), \rightarrow y_{1}=\phi_{2}^{*}(x), \\
& \phi_{1}\left(x, y_{2}\right)=\phi_{2}^{\prime}\left(x, y_{2}\right), \rightarrow y_{2}=\phi_{1}^{*}(x) .
\end{aligned}
$$

Finally, substituting the expressions in (36) in the leader's optimisation problem (1st) level, we end up with a single level convex optimisation problem, involving only the leader's optimisation variables, as follows:

$$
\begin{array}{ll} 
& \min _{x} f_{1}^{*}\left(x, y_{1}\left(x, y_{2}^{*}(x)\right), y_{2}\left(x, y_{1}^{*}(x)\right)\right), \\
\text { s.t. } & G_{1}\left(x, y_{1}\left(x, y_{2}^{*}\right), y_{2}\left(x, y_{1}^{*}\right)\right) \leq 0, \\
& x \in C_{r f}, \\
& C_{r f}=\left\{x \in X: \exists_{y_{1}, y_{2}} \in Y, Z, G_{2}\left(x, y_{1}, y_{2}\right) \leq 0, G_{3}\left(x, y_{1}, y_{2}\right) \leq 0\right\} .
\end{array}
$$

The algorithm is summarised in Table 3 and is illustrated with the following example.

\subsubsection{Illustrative example 2}

Consider the following linear bilevel programming example involving three followers at the second level (Anandalingman 1988): 
Table 3 Parametric programming algorithm for bilevel programming problems with multi-followers

\begin{tabular}{ll}
\hline Step & Description \\
\hline 1 & $\begin{array}{l}\text { Recast each of the subproblems in the lower } \\
\text { level as a multi-parametric programming } \\
\text { problem, with the variables out of their con- } \\
\text { trol being the parameters }(32,33) \\
\text { Solve the resulting problems using the suit- } \\
\text { able multi-parametric programming algo- } \\
\text { rithm } \\
\text { Compute a Nash equilibrium point by direct } \\
\text { comparison of the rational reaction sets (35) } \\
\text { Substitute each of the } K \text { solutions in the } \\
\text { leader's problem, and formulate the } K \text { one } \\
\text { level optimisation problems } \\
\text { Compare the } K \text { optima points and select the } \\
\text { best one }\end{array}$ \\
\hline
\end{tabular}

$$
\begin{gathered}
\min _{x, y_{1}, y_{2}, y_{3}} F\left(x, y_{1}, y_{2}, y_{3}\right)=-x-y_{1}-2 y_{2}-y_{3}, \\
\text { s.t. } \min _{y_{1}} f_{1}\left(x, y_{1}, y_{2}, y_{3}\right)=x-3 y_{1}+y_{2}+y_{3}, \\
\min _{y_{2}} f_{2}\left(x, y_{1}, y_{2}, y_{3}\right)=x+y_{1}-3 y_{2}+y_{3}, \\
\min _{y_{3}} f_{3}\left(x, y_{1}, y_{2}, y_{3}\right)=x+y_{1}+y_{2}-3 y_{3}, \\
\text { s.t. } \quad 3 x+3 y_{1} \leq 30, \\
2 x+y_{1} \leq 20, \\
y_{2} \leq 10 \\
y_{2}+y_{3} \leq 15, \\
y_{3} \leq 10, \\
x+2 y_{1}+2 y_{2}+y_{3} \leq 40, \\
x, y_{1}, y_{2}, y_{3} \geq 0 .
\end{gathered}
$$

Assume that the leader imposes all constraints to all followers. Thus, performing the steps described in Table 3:

Step 1. Recast optimisation subproblems $\min _{y_{1}} f_{1}, \min _{y_{2}} f_{2}$ and $\min _{y_{3}} f_{3}$ as multiparametric programming problems, with parameters being the set of variables out of their control.

Step 2. Solve the three multi-parametric programming problems using a suitable algorithm (Dua et al. 2002).

Follower 1

$$
C R_{1}^{1}\left\{\begin{array} { l } 
{ y _ { 1 } = - x + 1 0 , } \\
{ 0 \leq x , y _ { 2 } , y _ { 3 } \leq 1 0 , } \\
{ y _ { 2 } + y _ { 3 } \leq 1 5 , } \\
{ - 0 . 5 x + y _ { 2 } + 0 . 5 y _ { 3 } \leq 1 0 , }
\end{array} \quad C R _ { 1 } ^ { 2 } \left\{\begin{array}{l}
y_{1}=-0.5 x-y_{2}-0.5 y_{3}+20, \\
0 \leq x \\
0.5 x-y_{2}-0.5 y_{3} \leq-10 \\
y_{2} \leq 10 \\
y_{2}+y_{3} \leq 15
\end{array}\right.\right.
$$


Follower 2

$$
\begin{gathered}
C R_{2}^{1}\left\{\begin{array} { l } 
{ y _ { 2 } = 1 0 , } \\
{ 0 \leq x , y _ { 1 } , y _ { 3 } , } \\
{ x + y _ { 1 } \leq 1 0 , } \\
{ y _ { 3 } \leq 5 , } \\
{ 0 . 5 x + y _ { 1 } + 0 . 5 y _ { 3 } \leq 1 0 , }
\end{array} \quad C R _ { 2 } ^ { 2 } \left\{\begin{array}{l}
y_{2}=-y_{3}+15 \\
0 \leq x, y_{1} \\
x+y_{1} \leq 10 \\
5 \leq y_{3} \leq 10 \\
0.5 x+y_{1}-0.5 y_{3} \leq 5
\end{array}\right.\right. \\
C R_{2}^{3}\left\{\begin{array}{l}
y_{2}=-0.5 x-y_{1}-0.5 y_{3}+20 \\
0 \leq x, \\
x+y_{1} \leq 10, \\
-0.5 x-y_{1}+0.5 y_{3} \leq-5, \\
-0.5 x-y_{1}-0.5 y_{3} \leq-10
\end{array}\right.
\end{gathered}
$$

Follower 3

$$
\begin{gathered}
C R_{3}^{1}\left\{\begin{array} { l } 
{ y _ { 3 } = 1 0 , } \\
{ 0 \leq x , y _ { 1 } , y _ { 2 } , } \\
{ x + y _ { 1 } \leq 1 0 , } \\
{ y _ { 1 } \leq 5 , } \\
{ 0 . 5 x + y _ { 1 } + y _ { 2 } \leq 1 5 , }
\end{array} \quad C R _ { 3 } ^ { 2 } \left\{\begin{array}{l}
y_{3}=-y_{1}+15 \\
0 \leq x, y_{2}, \\
x+y_{1} \leq 10 \\
5 \leq y_{1}, \\
0.5 x+0.5 y_{1}+y_{2} \leq 12.5
\end{array}\right.\right. \\
C R_{3}^{3}\left\{\begin{array}{l}
y_{3}=-x-2 y_{1}-2 y_{2}+40, \\
0 \leq x, y_{1}, \\
x+y_{1} \leq 10, \\
-0.5 x-0.5 y_{1}-y_{2} \leq-12.5 \\
0.5 x+y_{1}+y_{2} \leq 20, \\
-0.5 x-y_{1}-y_{2} \leq-15 .
\end{array}\right.
\end{gathered}
$$

Step 3. Compute the Nash equilibrium point, through direct comparison of the explicit analytical rational reaction sets (39)-(41). Through this comparison we generate 18 regions, from which, 12 have empty feasible sets. After removing empty regions:

$$
\begin{gathered}
C R^{1}\left\{\begin{array}{l}
y_{1}=-x+10, \\
y_{2}=10, \\
y_{3}=x,
\end{array}\right. \\
C R^{3}\left\{\begin{array} { l } 
{ y _ { 1 } = - x + 1 0 , } \\
{ y _ { 2 } = - 0 . 5 x - y _ { 1 } - 0 . 5 y _ { 3 } + 2 0 , } \\
{ y _ { 3 } = - x - 2 y _ { 1 } - 2 y _ { 2 } + 4 0 , }
\end{array} \quad C R ^ { 4 } \left\{\begin{array}{l}
y_{1}=-x+10, \\
y_{2}=-y_{3}+15, \\
y_{3}=-x-2 y_{1}-2 y_{2}+40,
\end{array}\right.\right. \\
C R^{5}\left\{\begin{array}{l}
y_{1}=-0.5 x-y_{2}-0.5 y_{3}+20, \\
y_{2}=10, \\
y_{3}=-x-2 y_{1}-2 y_{2}+40, \\
y_{2}=-y_{3}+15, \\
y_{3}=-x-2 y_{1}-2 y_{2}+40,
\end{array}\right.
\end{gathered}
$$


For the sake of brevity we omit here the constraints for each critical region.

Step 4. Incorporate the expressions (42) into $F$, and formulate 6 single level convex optimisation problems. They result in the same unique solution, as follows:

$$
F=-35 ; \quad x=5 ; \quad y_{1}=5 ; \quad y_{2}=10 ; \quad y_{3}=5 .
$$

The global optimum found is identical to the one reported in Anandalingman (1988).

\section{An application to optimal control of multilevel systems}

An important application of the proposed theory is the hierarchical control of dynamic systems (Başar and Selbuz 1979), as shown in Fig. 3.

In hierarchical control, the performance of a dynamic system is optimised within a complex structure with different objective functions at different levels, for instance as shown in Fig. 3 for a control structure involving two levels. In such a system, typically described by a discrete-time dynamic model:

$$
x_{n+1}=A_{n} \cdot x_{n}+B_{n}^{0} \cdot u_{n}+\sum_{i=1}^{m} B_{n}^{i} \cdot v_{n}^{i},
$$

we have a central controller, the leader, and $m$ peripheral (local) controllers; $x_{n}$ is the state vector of the system, $u_{n}$ is the control vector of the central controller and $v_{n}^{i}$ is the control vector of the $i$ th local controller, all at time step $n$. Each local controller may have its own dynamics, which can be incorporated in Equation (43) (Başar and Selbuz 1979).

The goal is the optimisation of a quadratic objective function corresponding to the central controller:

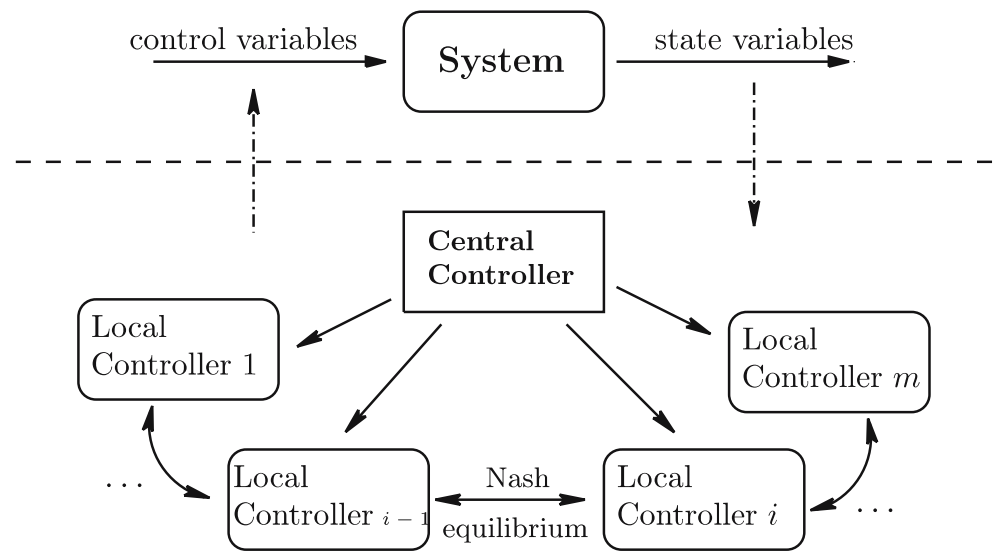

Fig. 3 Schematic representation of a hierarchical control configuration for a dynamic system 


$$
J_{0}=\left(x_{N}\right)^{T} Q_{N}^{0} x_{N}+\sum_{n=0}^{N-1}\left[\left(x_{n}\right)^{T} Q_{n}^{0} x_{n}+\left(u_{n}\right)^{T} R_{n}^{00} u_{n}+\sum_{i=1}^{m}\left(v_{n}^{i}\right)^{T} R_{n}^{0 i} v_{n}^{i}\right],
$$

subject to the optimisation of each local controller's objective function:

$$
J_{i}=\left(x_{N}\right)^{T} Q_{N}^{i} x_{N}+\sum_{n=0}^{N-1}\left[\left(x_{n}\right)^{T} Q_{n}^{i} x_{n}+\left(u_{n}\right)^{T} R_{n}^{i 0} u_{n}+\sum_{k=1}^{m}\left(v_{n}^{k}\right)^{T} R_{n}^{i k} v_{n}^{k}\right]
$$

Expressions (43), (44) and (45) give rise to a multi-level optimisation problem formulation: the leader, central controller, has control over the complete set of optimisation variables, whereas the local controllers have access to their own optimisation set, $v_{n}^{i}$, and corresponding objective function. The aim is to obtain the global optimum for the central controller and optimal strategies for the local controllers. Here, we consider the general case involving constraints (where most previews strategies considered the unconstrained case—see (Cruz 1978; Başar and Selbuz 1979; Başar and Olsder 1982)).

We seek an optimal policy, as follows:

$$
\begin{aligned}
\left\{u_{n}\right\}^{*} & =\left\{u_{0}^{*}, u_{1}^{*}, \ldots, u_{N}^{*}\right\} \rightarrow \gamma_{0}^{*}, \gamma_{0}^{*} \in \Gamma^{0}, \\
\left\{v_{n}^{1}\right\}^{*} & =\left\{\left(v_{0}^{1}\right)^{*},\left(v_{1}^{1}\right)^{*}, \ldots,\left(v_{N}^{1}\right)^{*}\right\} \rightarrow \gamma_{1}^{*}, \gamma_{1}^{*} \in \Gamma_{1}, \\
& \vdots \\
\left\{v_{n}^{i}\right\}^{*} & =\left\{\left(v_{0}^{i}\right)^{*},\left(v_{1}^{i}\right)^{*}, \ldots,\left(v_{N}^{i}\right)^{*}\right\} \rightarrow \gamma_{i}^{*}, \gamma_{i}^{*} \in \Gamma_{i}, \\
& \vdots \\
\left\{v_{n}^{m}\right\}^{*} & =\left\{\left(v_{0}^{m}\right)^{*},\left(v_{1}^{m}\right)^{*}, \ldots,\left(v_{N}^{m}\right)^{*}\right\} \rightarrow \gamma_{m}^{*}, \gamma_{2}^{*} \in \Gamma_{m} .
\end{aligned}
$$

Then the hierarchical control problem can be recast as the following multi-level constrained optimisation problem:

$$
\begin{array}{r}
\min _{\gamma_{0}, \gamma_{1}, \ldots, \gamma_{m}} J_{0}\left(\gamma_{0}, \gamma_{1}, \ldots, \gamma_{m}\right), \\
\text { s.t. } g_{1}\left(\gamma_{0}, \gamma_{1}, \ldots, \gamma_{m}\right) \leq 0, \\
\ldots,\left\{\begin{array}{c}
\min _{\gamma_{i}} J_{i}\left(\gamma_{0}, \gamma_{1}, \ldots, \gamma_{m}\right) \\
\text { s.t. } g_{2}^{i}\left(\gamma_{0}, \gamma_{1}, \ldots, \gamma_{m}\right), \leq 0
\end{array}\right\}, \ldots \text { (m local controllers). }
\end{array}
$$

Using Eq. (43) it is possible to express each state variable as a function of the initial state and the control decisions (Pistikopoulos et al. 2000). Therefore, $J_{0}$ and $J_{i}$ become functions only of the initial state: $J_{0}, J_{i}=f\left(x_{0}, \gamma_{1}, \gamma_{2}, \ldots, \gamma_{m}\right), \forall_{i} \in\{1,2, \ldots, m\}$. 
Since in the lower level of this two-level optimisation problem there are multiple optimisation subproblems, and there is the need to coordinate such group, it is fairly natural to assume a Nash equilibrium (Başar and Selbuz 1979):

$$
\begin{aligned}
& J_{1}\left(\gamma_{1}^{*}, \ldots, \gamma_{m}^{*}\right) \leq J_{1}\left(\gamma_{1}^{*}, \gamma_{2}, \gamma_{3}^{*}, \ldots, \gamma_{K}^{*}\right), \quad \forall_{\gamma_{1}} \in \Gamma_{1}, \\
& J_{2}\left(\gamma_{1}^{*}, \ldots, \gamma_{m}^{*}\right) \leq J_{1}\left(\gamma_{1}^{*}, \gamma_{2}, \gamma_{3}^{*}, \ldots, \gamma_{K}^{*}\right), \quad \forall_{\gamma_{2}} \in \Gamma_{2}, \\
& \quad \vdots \\
& J_{m}\left(\gamma_{1}^{*}, \ldots, \gamma_{m}^{*}\right) \leq J_{0}\left(\gamma_{1}, \gamma_{2}^{*}, \ldots, \gamma_{m-1}^{*}, \gamma_{m}^{*}\right), \quad \forall \gamma_{m} \in \Gamma_{m},
\end{aligned}
$$

where $\forall_{\gamma_{0}} \in \Gamma^{0}$ and $\forall_{x_{0}} \in X_{0}$, with $X_{0}$ being the feasible set of the system's initial state.

Problem (47) corresponds to a bilevel programming problem with multi-followers; the followers being the local controllers and the leader, the central controller. In contrast to Problem (31), the decisions involved in each subproblem are not only parametric relatively to the decisions of the remaining subproblems, but also depend on the initial state of the system. We refer to this class as multi-level optimisation problems with uncertainty. The algorithm in Table 3 can be directly applied to solve (47) only with a modification in Step 4, which requires 'the formulation and solution of $K$ multiparametric programming problems'.

A similar strategy can also be applied to tri-level optimisation problems. Moreover, if different models are involved in the subproblem, the proposed optimisation strategy is still applicable, with all control subproblems treated in a decentralised fashion. In the next section, a dynamic three person control system is described to illustrate the potential of the proposed approach.

\subsection{Illustrative example 3}

Consider a system which has a discrete dynamic behaviour described by the following linear state transition model (Nie et al. 2006):

$$
\begin{aligned}
& x_{t+1}=x_{t}+u_{t}-2 v_{t}^{1}+v_{t}^{2}, \\
& y_{t+1}^{1}=y_{t}^{1}+2 v_{t}^{1}, \\
& y_{t+1}^{2}=y_{t}^{2}+2 v_{t}^{2},
\end{aligned} \quad t=0,1,2,
$$

where $u, v^{1}$ and $v^{2}$ are input variables, and $x, y^{1}$ and $y^{2}$ output variables. And, with constraints on the input and state variables as follows:

$$
\begin{aligned}
& -30 \leq v_{t}^{1}, v_{t}^{2} \leq 30, \\
& -20 \leq u_{t} \leq 20, \\
& -10 \leq x_{0}, y_{0}^{1}, y_{0}^{2} \leq 10 .
\end{aligned} \quad t=0,1,2
$$


(a)

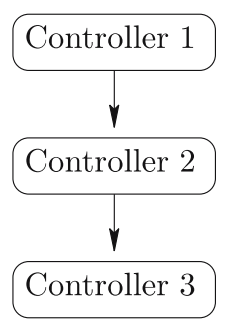

Three-level controller structure (b)

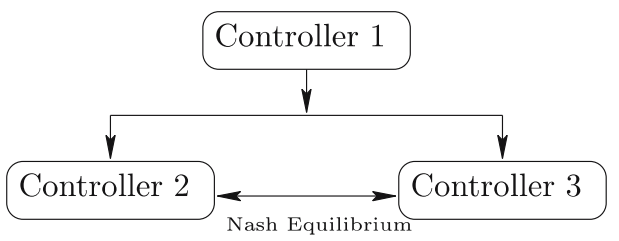

Multifollower controller structure

Fig. 4 Three-controller multilevel problem

Additionally, consider a three-controller system (Nie et al. 2006):

$$
\begin{aligned}
& \mathbf{J}_{\mathbf{1}}=\min _{u_{0}, u_{1}, u_{2}} 4 x_{3}+3 y_{3}^{1}+2 y_{3}^{2}+\sum_{t=0}^{2}\left\{\left(u_{t}\right)^{2}+\left(v_{t}^{1}\right)^{2}-\left(v_{t}^{2}\right)^{2}+2 u_{t} x_{t}+x_{t}^{2}\right\}, \\
& \mathbf{J}_{\mathbf{2}}=\min _{v_{0}^{2}, v_{1}^{2}, v_{2}^{2}} 2 x_{3}+3 y_{3}^{2}+\sum_{t=0}^{2}\left\{2 \cdot u_{t} v_{t}^{2}+\left(v_{t}^{1}+1\right)^{2}+\left(v_{t}^{2}+1\right)^{2}\right\}, \\
& \mathbf{J}_{3}=\min _{v_{0}^{1}, v_{1}^{1}, v_{2}^{1}} x_{3}+2 y_{3}^{1}-10 y_{3}^{2}+\sum_{t=0}^{2}\left\{-15 u_{t}+\left(v_{t}^{1}-1\right)^{2}-2 v_{t}^{1} v_{t}^{2}+\left(v_{t}^{2}\right)^{2}\right\},
\end{aligned}
$$

where $J_{1}, J_{2}$ and $J_{3}$ correspond to Controllers 1,2 and 3 , respectively. Figure 4 displays two possible configurations for the control structure of the considered system.

The objective is then to derive suitable optimal strategies for the two controller structures. Case (a) of Fig. (4) corresponds to a three-level optimisation problem, whereas case (b) refers to a bilevel multi-follower optimisation problem. Therefore, using the proposed methodology, fully implemented in Matlab $\AA$, we obtain the results summarised in Tables 4 and 5.

Table 4 Solution to the three-level optimisation problem

\begin{tabular}{llll}
\hline Critical region 1 & Critical region 2 & Critical region 3 & Critical region 4 \\
\hline$u_{0}=6.84615-0.76928 x_{0}$ & $u_{0}=-0.333333-1.8519 x_{0}$ & $u_{0}=-1.53333-1.6889 x_{0}$ & $u_{0}=-9-0.72732 x_{0}$ \\
$u_{1}=-20$ & $u_{1}=-1.33333+2.8148 x_{0}$ & $u_{1}=8.26667+1.5111 x_{0}$ & $u_{1}=20$ \\
$u_{2}=15.2308+0.15388 x_{0}$ & $u_{2}=-2-2.4444 x_{0}$ & $u_{2}=-20$ & $u_{2}=-20$ \\
$-10 \leq x_{0} \leq-6.63161$ & $-6.63161 \leq x_{0} \leq 7.36377$ & $7.36377 \leq x_{0} \leq 7.76466$ & $7.76466 \leq x_{0} \leq 10$ \\
\hline$v_{0}^{1}=v_{0}^{2}=-2-0.5 u_{0} ; v_{1}^{1}=v_{1}^{2}=-2-0.5 u_{1} ; v_{2}^{1}=v_{2}^{2}=-2-0.5 u_{2}$ &
\end{tabular}


Table 5 Solution to multi-follower problem

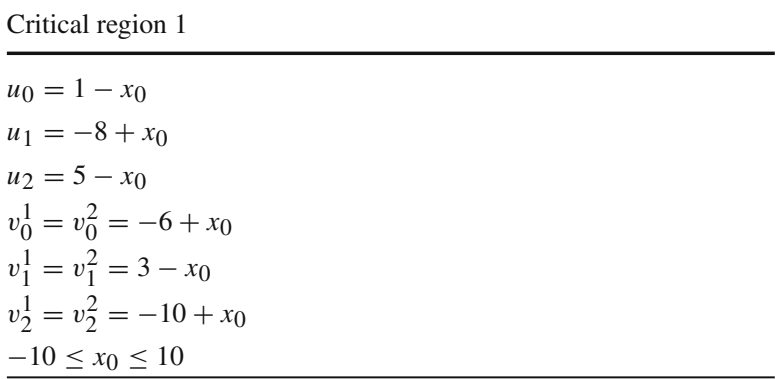

\section{Concluding remarks}

We have described a novel global optimisation strategy for the solution of hierarchical multi-level and decentralised multi-level programs based on our recent developments in multi-parametric programming theory and algorithms (Pistikopoulos et al. 2007a,b). The algorithms proposed are suitable for problems involving general convex objective functions and convex sets of constraints.

Current research focus is towards general non-linear models, for which recent results on global multi-parametric programming (Dua et al. 2004) can be used; and general dynamic multi-level problems, for which a dynamic programming approach coupled with parametric programming can be applied (Faísca et al. 2007a).

Acknowledgments Financial support from EPSRC (GR/T02560/01) and Marie Curie European Project PRISM (MRTN-CT-2004-512233) is gratefully acknowledged.

\section{References}

Anandalingman G (1988) A mathematical programming model of decentralized multi-level systems. J Opl Res Soc 39:1021-1033

Başar T (1975) Equilibrium solutions in two-person quadratic decision problems with static information structures. IEEE Trans Automatic Control 20(3):320-328

Başar T (1978) Decentralized multicriteria optimization of linear stochastic systems. IEEE Trans Automatic Control 23(2):233-243

Başar T, Olsder GJ (1982) Dynamic Noncooperative game theory. Academic, London

Başar T, Selbuz H (1979) Closed-loop Stackelberg strategies with applications in the optimal control of multilevel systems. IEEE Trans Automatic Control 24(2):166-179

Choi SC, Desarbo WS, Harker PT (1990) Product positioning under price competition. Manage Sci 36(2):175-199

Clark PA (1983) Embedded optimization problems in chemical process design. PhD Thesis. Department of Chemical Engineering, Carnegie-Mellon University, Pittsburg

Cruz JB (1978) Leader-follower strategies for multilevel systems. IEEE Trans Automatic Control 23(2):244-255

Dua V, Papalexandri KP, Pistikopoulos EN (2004) Global optimization issues in multiparametric continuous and mixed-integer optimization problems. J Global Optim 30:59-89

Dua V, Bozinis NA, Pistikopoulos EN (2002) A multiparametric programming approach for mixed-integer quadratic engineering problems. Comput Chem Eng 26:715-733

Faísca NP, Kouramas KI, Pistikopoulos EN (2007a) Dynamic programming, Chap 7. Wiley-VCH. Weinheim 
Faísca NP, Dua V, Saraiva PM, Rustem B, Pistikopoulos EN (2007b) Parametric global optimisation for bilevel programming. J Global Optim (in Press)

Fiacco AV(1976) Sensitivity analysis for nonlinear programming using penalty methods. Math Programm 10:287-311

Fiacco AV (1983) Introduction to sensitivity and stability analysis in nonlinear programming. Academic, New York

Floudas CA (2000) Deterministic global optimization. Kluwer, Dordrecht

Li M, Cruz JB, Simaan MA (2002) An approach to discrete-time incentive feedback stackelberg games. IEEE Trans Syst Man Cybern A: Syst Humans 32(4):472-481

Liu B (1998) Stackelberg-Nash equilibrium for multilevel programming with multiple followers using genetic algorithms. Comput Math Appl 36(7):79-89

McCormick GP (1976) Optimality criteria in nonlinear programming. SIAM-AMS Proc 1975(9):27

Morari M, Arkun Y, Stephanopoulos G (1980) Studies in the synthesis of control structures for chemical processes. AIChE J 26:220-232

Nie P, Chen L, Fukushima M (2006) Dynamic programming approach to discrete time dynamic feedback stackelberg games with independent and dependent followers. Euro J Oper Res 169:310-328

Pistikopoulos EN, Georgiadis MC, Dua V (2007a) Multi-parametric programming: theory, algorithms, and applications, vol 1. Wiley-VCH, Weinheim

Pistikopoulos EN, Georgiadis MC, Dua V (2007b) Multi-parametric model-based control: theory and applications, vol 2. Wiley-VCH, Weinheim

Pistikopoulos EN, Dua V, Bozinis NA, Bemporad A, Morari M (2000) On-line optimization via off-line parametric optimization tools. Comput Chem Eng 24:183-188

Rodić AD, Vukobratović MK (1999) Contribution to the integrated control synthesis of road vehicles. IEEE Trans Control Syst Technol 7(1):64-78

Ruan GZ, Wang SY, Yamakamoto Y, Zhu SS (2004) Optimality conditions and geometric properties of linear multilevel programming problem with dominated objective functions. J Optim Theory Appl 123(2):409-429

Shih HS, Wen UP, Lee ES, Lan KM, Hsiao HC (2004) A neural network approach to multiobjective and multilevel programming problems. Comput Math Appl 48:95-108

Stephanopoulos G, Ng C (2000) Perspectives on the synthesis of plant-wide control structures. J Process Control 10:97-111

Tolwinski B (1981) Closed-loop stackelberg solution to a multistage linear-quadratic game. J Optim Theory Appl 34(4):485-501

Vicente LN (1992) Bilevel programming. Master's Thesis. Department of Mathematics, University of Coimbra, Coimbra

Vicente LN, Calamai PH (1994) Bilevel and multilevel programming: a bibliography review. J Global Optim 5(3):291-306 